\title{
Fourniture par le sol et utilisation de l'azote minéral dans les prairies de montagnes volcaniques
}

\author{
P. Loiseau avec la collaboration technique de G. Merle, M. Rousseau et G. Bielicki \\ INRA, station d'agronomie, laboratoire des productions fourragères, 12 avenue du Brézet 63039 Clermont-Ferrand, Cedex, France
}

(reçu le 16 mai 1989, accepté le 1er octobre 1989)

Résumé - Les flux d'azote entre le sol et le peuplement sont étudiés dans 3 prairies de montagne développées sur andosol entre 1000 et $1350 \mathrm{~m}$ d'altitude et choisies pour leurs niveaux d'intensification contrastés. Les quantités d'azote récoltées en l'absence d'apports azotés et de légumineuses diminuent avec le nombre d'années de fauche sans restitution d'azote. Elles sont en moyenne de 29,75 et $100 \mathrm{~kg} \cdot \mathrm{ha}^{-1} \cdot \mathrm{an}^{-1}$ pour la pelouse pauvre $(P)$, la pelouse intensifiée $(\mathrm{M})$ et la prairie $(\mathrm{R})$. Les coefficients apparents d'utilisation des apports azotés mesurés après 5 ans de fauche augmentent de la pelouse à la prairie, avec respectivement $32,4,26,1$ et 51,7 pour $P$, $M$ et $R$. L'azote récolté dans les 3 sites obéit à une même loi exponentielle de réponse à l'azote total disponible, fourni par le sol et par les apports minéraux. Les coefficients réels (CRU) et apparents (CAU) d'utilisation de l'azote sont mesurés sur des peuplements non appauvris par la fauche : les CAU annuels sont de 63 et $77 \%$, et les CRU de 25 et $32 \%$ sur $P$ et M. Les CAU relatifs aux apports saisonniers diminuent avec la date d'apport. La fourniture du sol en présence d'apports est de 71 (P) et $133 \mathrm{~kg} \mathrm{~N} \cdot \mathrm{ha}^{-1} \cdot \mathrm{an}^{-1}(\mathrm{M})$ si elle est mesurée par le marquage ${ }_{15} \mathrm{~N}$, au lieu de $33(\mathrm{P})$ et $67 \mathrm{~kg} \mathrm{~N} \cdot \mathrm{ha}^{-1} \cdot \mathrm{an}^{-1}(\mathrm{M}) \mathrm{si}$ elle est mesurée par la récolte en absence d'apport. La discussion porte sur l'existence d'une interaction vraie entre l'apport et la fourniture minérale en azote du sol et sur le chiffrage de la minéralisation brute in situ.

azote $-{ }_{15} \mathrm{~N}$ - fertilisation - minéralisation - prairie - montagne

Summary - Nitrogen derived from soil and mineral $\mathrm{N}$ uptake efficiency in mountain volcanic pastures. The nitrogen fluxes from soil to plant population were investigated in 3 mountain grasslands on andepts at $1000-1350 \mathrm{~m}$ asl, selected for their contrasting levels of intensification. The mean nitrogen uptakes in the absence of mineral $N$ fertilization and leguminous plants decrease with the number of years without nitrogen restitution (Fig. 1). The mean values were 29, 75 and $100 \mathrm{~kg} \mathrm{~N} \cdot \mathrm{ha}^{-1} \cdot \mathrm{an}^{-1}$ for the poor grassland $(P)$, the intensified grassland $(M)$ and the grassland $(R)$ respectively. The apparent efficiencies of the nitrogen fertilizer, measured in the 5th year, increased from $32.4(P)$ to $26.1(M)$ and 51.7 (R) (Tab. I). However, for all grasslands the nitrogen recoveries in the harvested parts conformed to the same exponential relation for the total available nitrogen supplied by the soil and by fertilization (Fig. 2). The real and apparent recoveries were measured on grasslands without previous exhaustion of $N$ by cutting : the apparent recoveries were $63(P)$ and $77 \%(M)$, and the real recoveries $25(P)$ and $32 \%(M)(T a b . ~ I I I)$. The apparent coefficients due to seasonal supply decreased with the date of supply : in the poor grasslands, annual efficiencies account for 39 and $12 \%$ respectively of the applications in June and July; in the intensified grassland, annual efficiencies account for 59, 30 and $7 \%$ respectively of the applications in May, June and August. The nitrogen uptake from the soil in the presence of mineral fertilization is $71(P)$ and $133 \mathrm{~kg} \mathrm{~N} \cdot \mathrm{ha}^{-1} \cdot \mathrm{year}^{-1}(M)$ as measured by ${ }_{15} \mathrm{~N}$, instead of $33(P)$ and 65 $(M)$ as measured in the absence of fertilization (Tab. IV). The discussion focuses on the possibility of a real added nitrogen interaction and the evaluation of the in situ gross mineralization.

nitrogen - ${ }_{15} \mathrm{~N}$ - efficiency - fertilizer - grassland - mountain - mineralization - ANI

\section{INTRODUCTION}

Des études antérieures (Loiseau et al., 1984) ont montré que l'intensification des pelouses de moyenne montagne par l'amélioration de la conduite du pâturage, la fertilisation complète et l'augmentation du chargement augmentait à long terme l'utilisation de l'azote provenant des apports de fèces. Un bilan sur la provenance de l'azote mobilisé dans la croissance montrait que l'intensification augmentait aussi les quantités d'azote provenant de la minéralisation du sol. Cela suggérait que l'intensification, en particulier par les apports azotés, pouvait à long terme améliorer la minéralisation de l'azote organique préalablement accumulé dans le sol. 
Une série d'investigations a été entreprise pour préciser la dynamique de l'azote dans les systèmes sol-peuplement prairial et les formes de stockage de l'azote dans les matières organiques. On s'est servi d'une gamme de prairies contrastées. Les résultats développés ici concernent :

- la mesure de l'azote fourni par le sol en absence d'apports azotés;

- l'étude de l'azote utilisé dans la croissance en fonction des apports minéraux azotés;

- la mesure des coefficients réels d'utilisation des apports minéraux azotés;

- la mesure de l'azote fourni par le sol en présence ou en absence d'apport de $\mathrm{N}$ minéral. Les résultats concernant le comportement dans le sol de l'azote apporté seront exposés ultérieurement.

\section{MATÉRIEL ET MÉTHODES}

\section{Sites prairiaux}

Les investigations ont été réalisées dans le Massif des Monts Dore (Puy de Dôme) sur 3 prairies développées sur andosols. Deux des sites concernent des écosystèmes pâturés dérivant d'un même état initial : un témoin resté à l'état de pelouse peu exploitée par le bétail et une pelouse identique à l'origine, mais intensifiée depuis 15 ans. La pelouse pauvre est à base de Nardus stricta L., et la pelouse améliorée à base de Festuca rubra L.. Pour certaines études, un $3^{\theta}$ site a été ajouté sous la forme d'une prairie permanente de fauche, à Dactylis glomerata L. située dans les mêmes conditions de sol mais développée après un passé agricole ancien de culture. Les trois écosystèmes correspondent à des intensifications et des productions croissantes. La pelouse pauvre est exploitée en pâturage extensif à raison de 0 à $300 \mathrm{~kg}$ poids vif.ha $\mathrm{h}^{-1}$ par saison d'herbe, et sans apports fertilisants; la pelouse intensifiée est exploitée en pâturage tournant sous de forts chargements de 1200 à $1500 \mathrm{~kg}$ poids vif.ha ${ }^{-1}$ par saison d'herbe et reçoit annuellement une fertilisation complète comprenant $80 \mathrm{~kg} \mathrm{~N} \cdot \mathrm{ha}^{-1} \cdot \mathrm{an}^{-1}$. La prairie naturelle de fauche est développée après abandon de la culture depuis plus de 50 ans; elle reçoit de l'ordre de $200 \mathrm{~kg} \mathrm{~N} \cdot \mathrm{ha}^{-1} \cdot \mathrm{an}^{-1}$, sous forme de fumure organique et minérale.

Les 3 sites sont proches, mais à des altitudes respectives de 1350,1200 et $1050 \mathrm{~m}$ pour la pelouse pauvre $(P)$, la pelouse intensifiée $(M)$ et la prairie de fauche (R). Les sols, tous des andosols, sont excessivement riches en matières organiques avec, sur les 5 premiers $\mathrm{cm}$, des teneurs de $55,1(\mathrm{P}), 33,5(\mathrm{M})$ et $21,0 \%(R)$, les $C / N$ étant de $13,8(P), 11,3(M)$ et 10,6 (R). Ces conditions sont en principe favorables à l'existence d'une surminéralisation d'azote en présence d'apports fertilisants $\mathrm{N}$.

\section{Mesure de l'utilisation de l'azote dans la récolte à partir de la fourniture du sol}

Sur les 3 sites, on a conduit pendant 5 ans des petites parcelles en fauche sans apport d'azote minéral et sans déjections animales. Au cours de l'épuisement en azote, différentes variantes combinent ou non, la restitution sous forme minérale du phosphore et du potassium exportés dans la récolte, et le désherbage par des hormones pour éliminer la fixation symbiotique d'azote par le trèfle blanc. Le rythme de coupe a été de 3 coupes par an pour la pelouse intensifiée et la prairie de fauche, et seulement de 2 coupes pour la pelouse pauvre.

\section{Réponse au niveau de fertilisation azotée}

Sur le dispositif précédent, un essai de doses d'azote avec 2 répétitions et $0,100,200$ et $300 \mathrm{~kg} \mathrm{~N} \cdot \mathrm{ha}^{-1} \cdot \mathrm{an}^{-1}$ sous forme de nitrate d'ammonium est réalisé, après 5 années d'épuisement, avec des apports en $\mathrm{P}_{2} \mathrm{O}_{5}$ et $\mathrm{K}_{2} \mathrm{O}$ non limitants. Les rythmes d'exploitation sont identiques pour les différentes doses : 2 coupes par an pour $(P)$ et 3 coupes pour $(M)$ et $(R)$. Les apports d'azote ont été réalisés en 3 fois, à raison de $45 \%$ avant la première coupe, $30 \%$ après la première coupe et $25 \%$ après la deuxième coupe.

\section{Mesure des coefficients réels d'utilisation de l'azote et de la contribution réelle du sol à l'azote exporté}

Un essai avec apport de $\mathrm{NH}_{4} \mathrm{NO}_{3}$ marqué sur les 2 ions avec un excès de ${ }_{15} \mathrm{~N}$ de $14,80 \%$ a été mis en place en $2^{\circ}$ année sur des peuplements non préalablement épuisés par la fauche sans restitution de N. Cet essai a été suivi pendant 3 ans. L'azote est apporté à 3 reprises en mai, juin et août sur $M$, et à 2 reprises en juin et juillet sur $P$, à raison de $50 \mathrm{~kg} \mathrm{~N} \cdot \mathrm{ha}^{-1}$ par apport. Un nombre de blocs égal au nombre de coupes et d'apports permet de chiffrer à chaque coupe la contribution d'un apport donné à l'azote exporté. Pour ce faire, tous les blocs reçoivent la même fertilisation azotée marquée à des dates et sur des blocs différents. Le marquage est réalisé sur des placettes de $0,5 \mathrm{~m}^{2}$, avec 3 répétitions, mais les récoltes sont groupées pour l'analyse ${ }_{15} \mathrm{~N}$. Les analyses ont été réalisées à la station de Laon sous la responsabilité de B. Mary, avec un spectromètre de masse VG/SIRA 9.

L'anneau de garde pour l'épandage de l'engrais est limité à $10 \mathrm{~cm}$ sur 3 côtés des blocs, et élargi à $40 \mathrm{~cm}$ sur un côté pour y effectuer les prélèvements de sol marqué.

\section{RÉSULTATS}

\section{Azote exporté provenant du sol}

L'azote exporté en l'absence d'apports d'engrais azotés diminue de la $1^{\text {re }}$ à la $5^{\theta}$ année de fauche successive, avec un léger pic correspondant à la $4^{\theta}$ année, plus favorable sur le plan climatique. Sur les parcelles désherbées avec destruction du trèfle blanc, la fourniture du sol à la production épigée varie entre 39 et $22 \mathrm{~kg} \mathrm{~N} \cdot \mathrm{ha}^{-1} \cdot \mathrm{an}^{-1}$ pour la pelouse pauvre, 76 et $54 \mathrm{~kg}$ pour la pelouse intensifiée et 115 et $68 \mathrm{~kg}$ pour la prairie de 
fauche (Fig. 1). Les quantités moyennes d'azote exporté ont été de 29,75 et $100 \mathrm{~kg} \mathrm{~N} \cdot \mathrm{ha}^{-1} \cdot \mathrm{an}^{-1}$ respectivement pour $P, M$ et $R$.

Fig. 1. Récolte d'azote en l'absence d'apports fertilisants azotés.

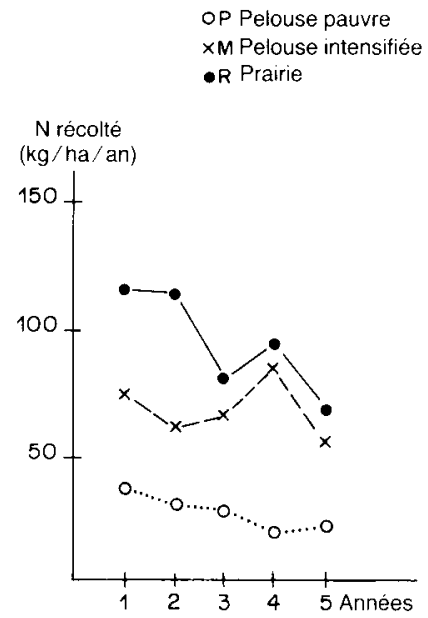

C'est sur la pelouse intensifiée, où le trèfle blanc était le plus abondant que la différence d'azote contenu dans la production a été la plus forte entre les traitements avec et sans désherbage. La participation de la fixation symbiotique à l'azote utilisé a été au maximum de $21 \mathrm{~kg}$ $\mathrm{N} \cdot \mathrm{ha}^{-1} \cdot \mathrm{an}^{-1}$ en première année, et a baissé par la suite en raison de la diminution du trèfle sous l'action du régime de fauche. L'influence de la restitution sous forme minérale du $P$ et du $K$ exportés par la récolte a été faible.

\section{Récolte d'azote en fonction des apports de $\mathbf{N}$ minéral, et coefficients apparents d'utilisa- tion (CAU)}

L'utilisation d'azote dans la production agricole en fonction des apports minéraux de $\mathrm{N}$ s'ajuste à une loi linéaire, différente pour chaque prairie et de la forme :

$$
\mathrm{N}_{\text {utilise }}=\mathrm{N}_{\mathrm{o}}+\left(\mathrm{CAU} \times \mathrm{N}_{\text {Apporte }}\right)
$$

L'ordonnée à l'origine, $N_{0}$, représente l'azote utilisé fourni par le sol. Dans le modèle il est supposé constant en fonction des apports et prend les valeurs respectives de $24,1,65,2$ et 70,8 pour les prairies P, M et $\mathrm{R}$ (Tableau I). La pente mesure le coefficient apparent d'utilisation des apports (CAU). Dans le modèle, elle est supposée constante pour les apports de 0 à $300 \mathrm{~kg} \mathrm{~N}$, et prend les valeurs respectives de 26,1,32,4 et $51,7 \%$ pour les peuplements $P, M$ et $R$ (Tableau I).

Les résidus des régressions linéaires précédentes montrent que la relation entre $\mathrm{N}$ exporté et $\mathrm{N}$ apporté est plutôt curviligne : concave pour
Tableau I. Corrélation linéaire entre azote utilisé et azote minéral apporté pour 3 prairies.

\begin{tabular}{|c|c|c|c|c|c|c|}
\hline \multirow[t]{2}{*}{ Praine } & \multirow{2}{*}{\multicolumn{2}{|c|}{$\begin{array}{l}\text { Constante }(\mathrm{No}) \\
(\mathrm{kg}) \mathrm{N}_{\mathrm{h}} \mathrm{ha}^{-1} \cdot \mathrm{an}^{-1}\end{array}$}} & \multicolumn{2}{|c|}{ Pente (CAU) } & \multirow[t]{2}{*}{$r^{2}$} & \multirow[t]{2}{*}{$d d^{-}$} \\
\hline & & Erreurst. & Valeur & $\begin{array}{l}\% \% \\
\text { Erreur st. }\end{array}$ & & \\
\hline$P$ & 24,1 & 5,0 & 26,1 & 2,7 & 0,94 & 7 \\
\hline$M$ & 65,2 & 7,5 & 32,4 & 4,0 & 0,91 & 7 \\
\hline R & 70,8 & 6,0 & 51,7 & 3,2 & 0,97 & 7 \\
\hline
\end{tabular}

la pelouse, concave puis rectiligne et même finalement convexe pour la prairie riche. On a donc recherché des modèles d'ajustement non linéaires. Les observations suivantes ont conduit à élaborer non pas un nouveau modèle par prairie mais un seul modèle non linéaire permettant un ajustement des 3 courbes de réponse. En effet, les différentes courbes semblent exprimer les premiers stades successifs d'une même relation sigmoïde; elles se superposent par une translation sur l'axe des apports $\mathbf{N}$ d'une valeur appelée CORR (Loiseau, 1989). Après cette translation, les 3 prairies obéissent à une même loi d'utilisation de l'azote dans la production en fonction d'un terme $\mathrm{N}_{\text {App }}+\mathrm{CORR}$, assimilable, à une constante près, à l'azote minéral total disponible, fourni par le sol et les apports $\left(N_{\text {Disp }}\right)$ (Fig. 2). II faut toutefois éliminer pour cela les points correspondant aux 2 doses les plus fortes pour la pelouse $\mathrm{M}$; car dans ce cas, il semble qu'un autre facteur que la fourniture d'azote minéral, lié à la structure du peuplement et notamment à la composition botanique vienne plafonner l'utilisation de l'azote.

$$
\begin{aligned}
& \text { - Pelouse pauvre (P) CORR }=0 \\
& \times \text { Pelouse intensifiée }(M) \text { CORR }=200 \\
& \text { - Prairie de fauche }(R) \text { CORR }=210
\end{aligned}
$$

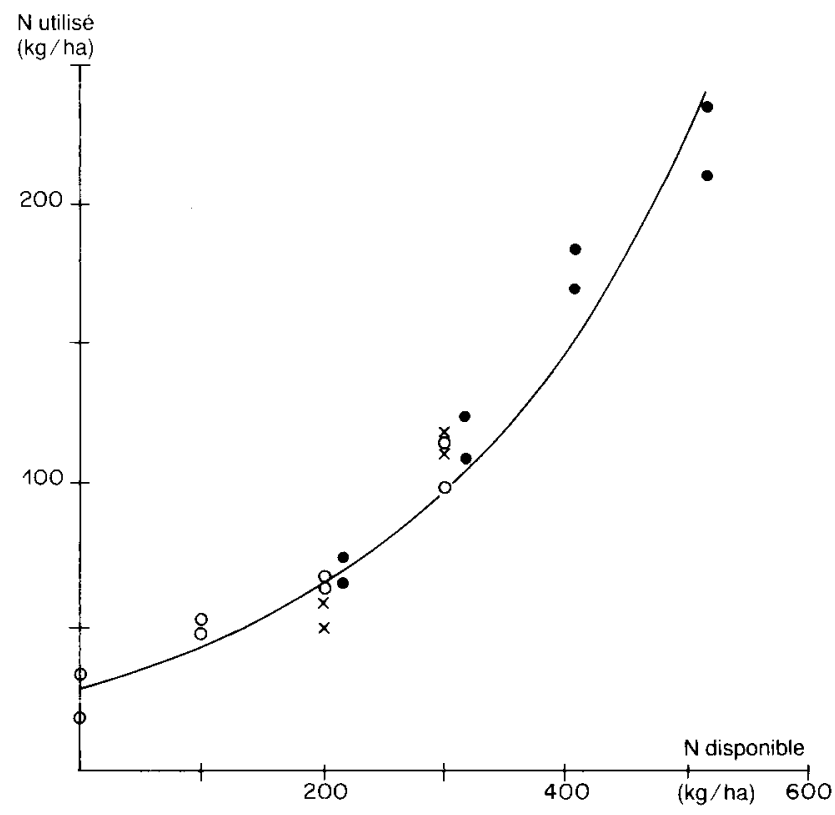

Fig. 2. Loi de réponse de 3 prairies à l'azote total disponible. 
Les coefficients CORR donnant la meilleure relation entre azote utilisé et azote disponible sont respectivement de 0,200 et $210 \mathrm{~kg}$ $\mathrm{N} \cdot \mathrm{ha}^{-1} \cdot \mathrm{an}^{-1}$ pour $\mathrm{P}, \mathrm{M}$ et $\mathrm{R}$. Ils représentent les valeurs des vecteurs translation qui permettent de faire coïncider les 3 courbes de réponse. Selon l'interprétation précédente, ils constituent une estimation minimale de la minéralisation brute d'azote dans le sol. La relation exponentielle calculée avec les résultats obtenus sur les 3 prairies est la suivante (Fig. 2) :

$$
\begin{gathered}
N_{\text {utilisé }}=N_{o} \exp \left(a \times N_{\text {Dispanible }}\right) \\
r^{2}=0,937 ; n_{0}=28 ; a=4,35 \times 10^{-3} ; \\
N_{\text {Disponible }}=\left(N_{\text {App }}+\text { CORR }\right),
\end{gathered}
$$

entre 0 et $550 \mathrm{~kg} \mathrm{~N} \cdot \mathrm{ha}^{-1} \cdot \mathrm{an}^{-1}$.

Selon cette relation, une valeur de $\mathrm{N}$ disponible nulle permet toujours une exportation de $28 \mathrm{~kg} \mathrm{~N} \cdot \mathrm{ha}^{-1} \cdot \mathrm{an}^{-1}$, qui pourrait correspondre, pour partie, à une mobilisation interne d'azote à partir des organes non récoltés du peuplement. La dérivée de la formule exprime le coefficient d'utilisation pour chaque $\mathrm{kg}$ successif d'azote disponible (Tableau II). Ce dernier est une fonction de la disponibilité déjà acquise : il augmente de 12 à $69 \%$ entre le premier et. le $400^{\theta} \mathrm{kg}$ de $\mathrm{N}$ minéral disponible, l'estimation devenant statistiquement moins fiable pour les disponibilités supérieures. II permet une interprétation des CAU moyens de l'azote apporté. La valeur constante de $26 \%$ obtenue précédemment pour le CAU moyen de la pelouse pauvre $(P)$ entre 0 et $300 \mathrm{~kg}$ d'apport $\mathrm{N}$ s'interprète alors comme une valeur moyenne correspondant à des CAU Marginaux de l'azote croissant de 12 à $45 \%$ entre les disponibilités de 0 à $300 \mathrm{~kg} \mathrm{~N}$. De même le CAU moyen de $52 \%$ obtenu pour la prairie riche (R) entre 0 et $300 \mathrm{~kg}$

Tableau II. Coefficients d'Utilisation moyens et margi-

\begin{tabular}{|c|c|c|c|c|c|c|c|c|c|}
\hline \multirow[b]{2}{*}{ Prainie } & \multirow[b]{2}{*}{$\begin{array}{l}\text { NApp. } \\
\mathrm{kgN} \\
\left(h a^{-1}\right)\end{array}$} & \multicolumn{2}{|c|}{ Expérimental } & & \multicolumn{2}{|l|}{ Modele } & & \multirow[b]{2}{*}{$\begin{array}{l}\text { CAU } \\
\text { marg. } \\
\%\end{array}$} \\
\hline & & $\begin{array}{l}\text { Expérim } \\
\text { Recolte } \\
\mathrm{kg} \mathrm{MS} \text {. } \\
\left(\mathrm{ha}^{-1}\right)\end{array}$ & $\begin{array}{l}\mathrm{kgN} . \\
\left(h^{-1}\right)\end{array}$ & $\begin{array}{l}\text { Linés } \\
\text { CAU } \\
\text { moy. } \\
(\%)\end{array}$ & $\begin{array}{l}\text { ire } \\
\text { CAU } \\
\text { calc. } \\
(\%)\end{array}$ & $\begin{array}{l}\text { NDisp. } \\
\mathrm{kg} \mathrm{N} \text {. } \\
\left(h \mathrm{a}^{-1}\right)\end{array}$ & $\begin{array}{l}\text { Exponer } \\
\text { Récolte } \\
\mathrm{kgN} \text {. } \\
\left(\mathrm{ha}^{-1}\right)\end{array}$ & $\begin{array}{l}\text { CAU } \\
\text { moy. } \\
\%\end{array}$ & \\
\hline $\bar{p}$ & 0 & 1267 & 26,3 & - & - & 0 & 28,0 & - & 12,2 \\
\hline$p$ & 100 & 2106 & 50 & 24,4 & 26,1 & 100 & 43,3 & 15,3 & 18,8 \\
\hline p & 200 & 2434 & 68,3 & 21,0 & 26,1 & 200 & 66,8 & 19,4 & 29,1 \\
\hline$p$ & 300 & 3414 & 107,3 & 27,0 & 26,1 & 300 & 103,3 & 26,5 & 44,9 \\
\hline$M$ & 0 & 2358 & 54,4 & - & - & 200 & 66,8 & - & 29,1 \\
\hline$M$ & 100 & 4194 & 114,8 & 60,4 & 32,4 & 300 & 103,3 & 36,5 & 44,9 \\
\hline$M$ & 200 & 4247 & 127,8 & 36,7 & 32,4 & 400 & $+32,4$ & 46,3 & 69,4 \\
\hline$M$ & 300 & 4789 & 158,0 & 34,5 & 32,4 & 500 & 246,5 & 59,9 & 107,2 \\
\hline $\mathrm{R}$ & 0 & 3813 & 72,1 & - & - & 210 & 69,5 & - & 30,2 \\
\hline 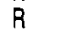 & 100 & 51 & 117 & 45,4 & 51,7 & 310 & 107,8 & 38,3 & 46,9 \\
\hline & 200 & 66 & 183 & 55,6 & 51,7 & 410 & 166,6 & 48,6 & 72,5 \\
\hline $\mathrm{P}$ & 0 & 774 & 223,6 & 50,5 & 51,7 & 510 & 257,4 & 62,6 & 112,0 \\
\hline
\end{tabular}
naux de l'azote : valeurs expérimentales, et selon le modèle exponentiel de réponse à l'azote disponible.

CAU moy. : Coefficient Apparent d'Utilisation de l'azote apporté.

CAU marg. : Coefticient Apparent Marginal d'Utilisation de l'azote dis. ponible à la disponibilité en azote considérée.

$N$ Disponible $=N$ Apporté + CORR, avec CORR $(P)=0$, $\operatorname{CORR}(M)=200$ et $\operatorname{CORR}(R)=210 \mathrm{~kg} \mathrm{~N} \mathrm{ha}{ }^{-4} \cdot \mathrm{an}^{-1}$. d'apport $\mathrm{N}$ correspond à des CAU Marginaux variant de 30 à $112 \%$ entre les disponibilités de 210 et $510 \mathrm{~kg} \mathrm{~N}$.

\section{Utilisation réelle des apports minéraux, et contribution de l'azote du sol à la production}

L'expérimentation avec marquage de l'apport minéral azoté permet la mesure des coefficients réels d'utilisation (CRU) des apports (Tableau III). L'année de l'apport, le CRU est de $25,5 \%$ sur la pelouse pauvre et $32,2 \%$ sur la pelouse intensifiée. L'engrais minéral est encore utilisé pendant les années suivantes, mais selon des taux plus faibles, qui portent les CRU sur 3 ans à $30,6 \%$ $(P)$ et $38,9 \%(M)$. Les CRU sont très inférieurs aux CAU. La valeur des CAU est de 63,5\% (P) et $77,2 \%$ (M) lors de l'année de l'apport pour les doses respectives de 100 (P) et $150 \mathrm{~kg}$ $\mathrm{N} \cdot \mathrm{ha}^{-1} \cdot \mathrm{an}^{-1}(\mathrm{M})$.

L'utilisation des apports dépend aussi de leurs dates : elle diminue pour les apports tardifs. Ainsi, les CRU sur 3 ans sont de $42 \%$ pour l'apport précoce de début juin et de $17 \%$ pour l'apport de juillet dans le cas de la pelouse pauvre. Pour la pelouse intensifiée, ils diminuent de 64 à 36 et $17 \%$ entre les apports de mai, juin et août.

La fourniture d'azote par le sol à la récolte est obtenue par différence entre l'azote total utilisé dans la production épigée et l'azote utilisé prove-

Tableau III. Coefficients réels et apparents d'utilisation

\begin{tabular}{|c|c|c|c|c|c|c|c|c|c|}
\hline $\begin{array}{l}\text { Pelouse pauvre ( } p \text { ) } \\
\text { Année } \\
\text { Date de récolte }\end{array}$ & $10 / 7$ & 1 & $4 / 10$ & $12 \pi$ & & $2 / 10$ & $\begin{array}{r}3 \\
8 / 7\end{array}$ & & $1-3$ \\
\hline $\begin{array}{l}\text { N App. (kg/ha) } \\
\text { N Exp. (kg/ha) } \\
\text { N Exp. (année) }\end{array}$ & $\begin{array}{l}49,2 \\
67,8\end{array}$ & $95,8^{2}$ & $\begin{array}{l}49,2 \\
27,9\end{array}$ & $\overline{12,4}$ & 427,4 & $4^{-}$ & $\begin{array}{l}- \\
19,4 \\
19,4\end{array}$ & 4 & $\begin{array}{l}98,4 \\
- \\
-\end{array}$ \\
\hline $\begin{array}{l}\text { CRU }(\%) \\
1^{\text {er }} \text { apport } \\
2^{\circ} \text { apport } \\
\text { Moyen }\end{array}$ & $\begin{array}{l}33,0 \\
-\quad 2\end{array}$ & 25,5 & $\begin{array}{r}6,1 \\
11,8\end{array}$ & $\begin{array}{l}1,4 \\
1,2\end{array}$ & $\begin{array}{l}4 \\
2 \\
2,9\end{array}$ & $\begin{array}{l}0,6 \\
2,6\end{array}$ & $\begin{array}{l}1,0 \\
1,2 \\
2,2\end{array}$ & $\begin{array}{ll}0 & 4 \\
2 & 1 \\
2 & 3\end{array}$ & $\begin{array}{l}42,0 \\
16,9 \\
30,6\end{array}$ \\
\hline $\begin{array}{l}N \operatorname{Exp} .(1) \\
-C A U(\%)\end{array}$ & & 33,2 & & & $\begin{array}{c}25,6 \\
-\end{array}$ & & & $\begin{array}{l}21,3 \\
-\end{array}$ & \\
\hline $\begin{array}{l}\text { Pelouse intensifiée ( } \\
\text { Année } \\
\text { Date de récolte }\end{array}$ & $20 / 6$ & $\begin{array}{r}1 \\
5 / 8\end{array}$ & $13 / 10$ & $25 / 6$ & $\begin{array}{r}2 \\
9 / 8\end{array}$ & $16 / 10$ & $26 / 5$ & $\begin{array}{l}3 \\
7 / 8\end{array}$ & $\begin{array}{l}1-3 \\
-\end{array}$ \\
\hline $\begin{array}{l}\text { N App. (kg/ha) } \\
\text { N Réc. (kg/ha) } \\
\text { N Exp (année) }\end{array}$ & $\begin{array}{l}49,2 \\
99,0\end{array}$ & $\begin{array}{r}49,2 \\
55,7 \\
181\end{array}$ & $\begin{array}{l}49,2 \\
26,3 \\
1,0\end{array}$ & $\overline{40,2}$ & $\overline{20,7 \overline{1}}$ & $\overline{13,4}$ & $\overline{48,0}$ & $\overline{25,4}$ & $\begin{array}{l}147,6 \\
\overline{-}, 4\end{array}$ \\
\hline $\begin{array}{c}\text { CRU (\%) } \\
\text { 1er apport } \\
2^{8} \text { apport } \\
3^{\theta} \text { apport } \\
\text { Moyen }\end{array}$ & & $\begin{array}{l}10,0 \\
26,4 \\
- \\
32,2\end{array}$ & $\begin{array}{l}2,3 \\
3,8 \\
7,1\end{array}$ & $\begin{array}{l}2,0 \\
2,9 \\
3,7\end{array}$ & $\begin{array}{l}0,4 \\
1,1 \\
3,0 \\
4,9\end{array}$ & $\begin{array}{l}0,4 \\
0,4 \\
0,7\end{array}$ & $\begin{array}{l}1,1 \\
1,2 \\
1,5\end{array}$ & $\begin{array}{l}0,3 \\
0,5 \\
0,7 \\
1,8\end{array}$ & $\begin{array}{l}63,6 \\
36,2 \\
16,8 \\
38,9\end{array}$ \\
\hline $\begin{array}{l}\text { NExp. }(1) \\
\text { CAU }(\%)\end{array}$ & & $\begin{array}{l}67,2 \\
77,2\end{array}$ & & & $\begin{array}{l}69,9 \\
-\end{array}$ & & $\begin{array}{l}85, \\
-\end{array}$ & 5,7 & - \\
\hline
\end{tabular}
des apports minéraux $\mathrm{N}$ sur la pelouse, selon son niveau d'intensification.

(1) Moyenne des chiffres obtenus la même année sur l'essai d'épuisement; N App. : azote minéral apporté; N Exp. : azote exporté. 
Tableau IV. Sources d'azote minéral utilisé.

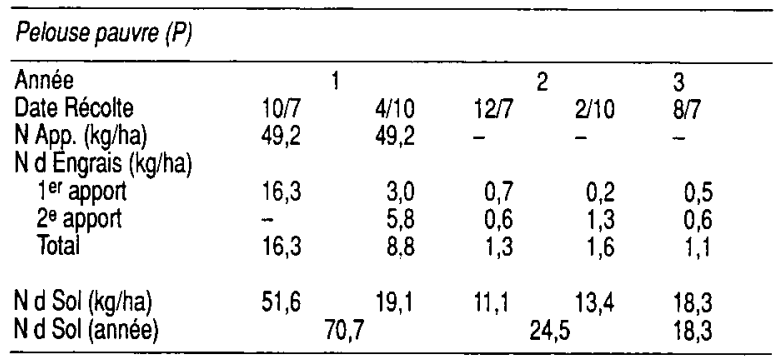

Pelouse intensifiée (M)

\begin{tabular}{llllllllll} 
Année & & 1 & & & 2 & & 3 \\
Date récolte & $20 / 6$ & $5 / 8$ & $13 / 10$ & $25 / 6$ & $9 / 8$ & $16 / 10$ & $26 / 5$ & $7 / 8$ \\
N App. (kg/ha) & 49,2 & 49,2 & 49,2 & - & - & - & - & - \\
N dEngrais (kg/ha) & & & & & & & & \\
$\quad$ 1er apport & 23,1 & 4,9 & 1,1 & 1,0 & 0,2 & 0,2 & 0,6 & 0,2 \\
2e apport & - & 13,0 & 1,9 & 1,4 & 0,5 & 0,2 & 0,6 & 0,3 \\
3e apport & - & - & 3,5 & 1,8 & 1,5 & 0,4 & 0,8 & 0,3 \\
$\quad$ Total & 23,1 & 17,9 & 6,5 & 4,2 & 2,2 & 0,8 & 1,9 & 0,8 \\
Nd Sol & 75,9 & 37,8 & 19,9 & 36,0 & 18,5 & 12,6 & 46,1 & 24,6 \\
Nd Sol (année) & & 133,5 & & & 67,1 & & & 70,7 & \\
\hline
\end{tabular}

$\mathrm{N} d$ Engrais : azote provenant de l'engrais; $\mathrm{N} \mathrm{d}$ Sol : azote provenant du sol.

nant de l'engrais (Tableau IV). L'année de l'apport, elle est de $70,7(P)$ et $133,5 \mathrm{~kg} \mathrm{~N} \cdot \mathrm{ha}^{-1}(\mathrm{M})$. Les années suivantes, la fertilisation est arrêtée et la fourniture du sol tombe à 24,5 et $18,3 \mathrm{~kg}$ $\mathrm{N} \cdot \mathrm{ha}^{-1}$ sur $\mathrm{P}$, et à 67,1 et $70,7 \mathrm{~kg} \mathrm{~N} \cdot \mathrm{ha}^{-1}$ sur $\mathrm{M}$. Ces chiffres sont du même ordre que ceux obtenus sur les petites parcelles d'épuisement pendant les mêmes années, soit 25,6 et $21,3(P)$; 69,9 et 85,7 (M) (Tableau III). On observe donc un effet important de l'apport minéral $\mathrm{N}$ sur la fourniture nette d'azote minéral par le sol. Le supplément de fourniture par le sol dû à l'apport (interaction ou ANI, Jenkinson et al., 1985) se chiffre à $37,5(P)$ et $66,3 \mathrm{~kg} \mathrm{~N} \cdot \mathrm{ha}^{-1} \cdot \mathrm{an}^{-1}(\mathrm{M})$ supplémentaires fournis par le sol l'année de l'apport, pour des apports respectifs de 100 et $150 \mathrm{~kg} \mathrm{~N}$ mineral $\cdot \mathrm{ha}^{-1} \cdot \mathrm{an}^{-1}$.

\section{DISCUSSION}

\section{Compétition sol-peuplement pour l'utilisation de l'azote}

La compétition pour l'utilisation de l'azote minéral entre le peuplement et le sol est reflétée par la forme initiale exponentielle de la courbe de réponse aux apports azotés : le coefficient marginal d'utilisation des apports supplémentaires est proportionnel à l'azote mobilisé initialement dans les parties récoltées :

$$
\mathrm{CAU}_{\mathrm{m}}=\mathrm{N}_{\mathrm{o}} \times a \exp \left(\mathrm{a} \times \mathrm{N}_{\mathrm{disp}}\right)
$$

Ainsi, les premiers $\mathrm{kg}$ d'azote disponibles sont surtout utilisés pour la production de racines ou pour la réorganisation dans le sol en général.
Ceci indique aux faibles fournitures une certaine prééminence de la croissance racinaire et (ou) de la demande du sol en azote sur la satisfaction des besoins en azote du peuplement pour la croissance épigée. Cette interprétation semble préférable à celle d'une colonisation insuffisante du sol par les racines expliquant la faible récolte d'azote. Une proportion élevée de la demande épigée est alors assurée par un pool d'azote interne au peuplement, annuellement utilisé au printemps et reconstitué par recyclage interne à la plante. Lorsque la fourniture de $\mathrm{N}$ augmente grâce aux apports ou à une minéralisation plus élevée, le supplément d'azote disponible est de mieux en mieux utilisé pour la croissance aérienne. Les doses employées sont insuffisantes pour voir apparaître un défaut de prélèvement dû à une saturation des capacités d'absorption du peuplement.

\section{Comparaison des CRU et des CAU. Interac- tion due à l'apport}

Les CAU mesurés sans épuisement préalable du sol sont élevés. Ils augmentent de $63 \%$ sur $P$ à $85 \%$ sur R (Tableau V), dans le même sens que les résultats de Cuttle (1983) : $32 \%$ sur sol tourbeux et $57 \%$ sur sol brun. Ils sont conformes aux données de la littérature : 55 à $80 \%$ (Ball, 1984), 79 à $95 \%$ pour des apports de printemps sur ray grass anglais (Dawson \& Ryden, 1985). En revanche, les CRU sont faibles : $25(P)$ et $32 \%$ (M) contre 51-59\% (Colbourn, 1983), 52\% (Whitehead \& Dawson, 1984), 52\% (Barraclough et al., 1985), 55\% (Bristow et al., 1987), tous sur ray grass anglais, et $55 \%$ sur fétuque élevée (Triboi, 1987).

Les mobilisations épigées d'azote en l'absence d'apports, de 29 (P) et $75(\mathrm{M}) \mathrm{kg} \mathrm{N} \cdot \mathrm{ha}^{-1} \cdot \mathrm{an}^{-1}$ sont similaires à celles obtenues par Cuttle (1983), sur des pâturages de même nature :

Tableau V. Comparaison des CAU et des CRU et influence de l'épuisement en azote.

\begin{tabular}{llll}
\hline $\begin{array}{l}\text { Chiffres obtenus l'année de l'apport } \\
\text { Site prairial }\end{array}$ & $p$ & $M$ & $A$ \\
\hline Sans épuisement (2e année). & & & \\
Apport N minéral (kg/ha) & $50+50$ & $50+50+50$ & $100+50+50$ \\
CAU (\%) & 63,5 & 77,2 & 85,2 \\
CRU (\%) & 25,5 & 32,2 & - \\
Cl (\%) & 38,0 & 45,0 & - \\
N sol (kg/ha) & 33,2 & 67,1 & 110,5 \\
Avec épuisement (6e année). & & & \\
Apport N minéral (kg/ha) & $45+30+25$ & $67+45+37$ & $90+60+50$ \\
CAU (\%) & 48,7 & 44,6 & 31,8 \\
N sol (kg/ha) & 17,3 & 58,1 & 67,7 \\
\hline
\end{tabular}

$\mathrm{N}$ sol : $\mathrm{N}$ récolté en l'absence d'apport; $\mathrm{Cl}$ : Coefficient d'interaction. $\mathrm{Cl}=\mathrm{CAU}-\mathrm{CRU}$. 
$11 \mathrm{~kg} \mathrm{~N} \cdot \mathrm{ha}^{-1} \cdot \mathrm{an}^{-1}$ sur un sol tourbeux et $66 \mathrm{~kg}$ sur un sol brun. On observe donc après intensification à la fois une fourniture accrue du sol et une augmentation des coefficients d'utilisation des apports.

La fourniture nette par le sol d'azote minéral à la récolte est plus élevée quand elle est estimée par le traceur que par le témoin non fertilisé. L'azote prélevé par la prairie peut provenir de 3 sources : une source issue du sol $\left(\mathrm{N}_{\text {sol }}\right)$, une source issue de l'engrais $\left(N_{\text {dff }}\right)$ et, éventuellement, une source supplémentaire par le sol, liée à l'apport minéral (interaction due à l'apport ou ANI, Jenkinson et al., 1985). Les deux effets de l'engrais, direct et indirect, sur l'azote de la récolte sont pris en compte par le CAU, alors que le seul effet direct est mesuré avec le CRU. On peut calculer par différence l'effet indirect de l'ap-

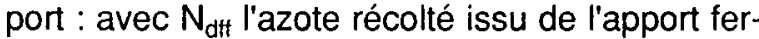
tilisant, en $\mathrm{kg} \cdot \mathrm{ha}^{-1}$, on a : NF = CRU $\times$ Apport, et $\mathrm{NF}+\mathrm{ANI}=\mathrm{CAU} \times$ Apport, d'où :

$$
A N I=(C A U-C R U) \times \text { Apport }
$$

On pourrait appeler coefficient d'interaction (CI), le terme CAU-CRU. II vaut $38,0 \%$ et $45,0 \%$ pour des apports respectifs de 100 et $150 \mathrm{~kg} \mathrm{~N}$ minéral sur $P$ et $M$ (Tableau $V$ ), contre $21 \%$ en moyenne pour les prairies de plaine sans forts excès de matières organiques (Machet et al., 1987), ou même 0 (Barraclough et al., 1985). II est supérieur à la fraction de l'engrais réorganisée mesurée 2 ans après l'apport, soit respectivement 33,8 et $36,6 \%$. Mais, en toute logique, il faut comparer les $\mathrm{Cl}$ à la fraction réorganisée au bout d'un an : celle-ci peut s'estimer en ajoutant aux chiffres précédents les CRU de la $2^{\mathrm{e}}$ année (2,9\% pour $P$ et $4,9 \%$ pour $M)$, soit $36,7 \%(P)$ et $41,5 \%$ (M). Observons-nous en montagne une interaction vraie (surminéralisation) ou apparente (échange de pools)? Malgré un $\mathrm{Cl}$ de $65 \%$, Dawson \& Ryden (1985) ne peuvent pas confirmer l'existence d'une réelle surminéralisation sous un RGA irrigué. De même Barraclough et al. (1985) rendent la seule substitution de pool par immobilisation et minéralisation responsable de I'ANI. Dans le cas présent, les $\mathrm{Cl}$ légèrement supérieurs à la fraction réorganisée dans le sol n'apportent, en absence de calcul statistique, qu'une faible présomption sur son existence.

Soit R l'azote exporté, O l'organisation, $\mathrm{M}$ la minéralisation brute et $P$ les pertes. Les indices $e$ et $s$ affectent l'azote issu de l'engrais et du sol. La notation ' concerne les flux en absence d'engrais. On a :

$$
\begin{aligned}
M & =O_{s}+R_{s}+P_{s} \\
M^{\prime} & =O_{s}^{\prime}+R_{s}^{\prime}+P_{s}^{\prime}
\end{aligned}
$$

L'ANI réelle s'écrit $A N I_{r}=M-M$, et l'ANI me- surée, sans présomption sur sa nature s'écrit $\mathrm{ANI}_{m}=\mathrm{R}_{s}-\mathrm{R}_{s}^{\prime}$.

$$
\begin{gathered}
\mathrm{ANI}_{r}=\left(\mathrm{O}_{s}-\mathrm{O}^{\prime} s\right)+\left(\mathrm{R}_{s}-\mathrm{R}_{s}{ }^{\prime}\right)+\left(\mathrm{P}_{s}-\mathrm{P}^{\prime}{ }_{s}\right) . \\
\mathrm{ANI}_{r}=\mathrm{ANI}_{m}+\left(\mathrm{O}_{s}-\mathrm{O}_{s}^{\prime}\right)+\left(\mathrm{P}_{s}-\mathrm{P}^{\prime}{ }_{s}\right)
\end{gathered}
$$

Si les pertes d'azote provenant du sol sont identiques en présence ou en absence d'apport on a:

$$
\mathrm{ANI}_{r}=\mathrm{ANI}_{m}+\left(\mathrm{O}_{s}-\mathrm{O}_{s}^{\prime}\right)
$$

Soit $O$ l'organisation totale $O=O_{s}+O_{\theta}$ et $\mathrm{O}^{\prime}=\mathrm{O}^{\prime}$

$$
\mathrm{ANI}_{r}=\mathrm{ANI}_{m}+\left(\mathrm{O}-\mathrm{O}^{\prime}\right)-\mathrm{O}_{e}
$$

L'ANI mesurée est de $66,3 \mathrm{~kg} \mathrm{~N} \cdot \mathrm{ha}^{-1} \cdot \mathrm{an}^{-1}$ pour $M(133,5$ moins 67,2$)$, et de 37,5 pour $P(70,7$ moins 33,2$)$. Comme indiqué ci-dessus, le terme $\mathrm{O}_{e}$ vaut $36,7 \%(\mathrm{P})$ et $41,5 \%$ (M) des apports, soit $36,1(P)$ et $61,3 \mathrm{~kg} \mathrm{~N} \cdot \mathrm{ha}^{-1} \cdot \mathrm{an}^{-1}(\mathrm{M})$. D'où :

$\mathrm{ANI}_{r}(\mathrm{P})=\left(\mathrm{O}-\mathrm{O}^{\prime}\right)+5,0$ et $\mathrm{ANI}_{r}(\mathrm{M})=\left(\mathrm{O}-\mathrm{O}^{\prime}\right)+$ 1,4 .

Ainsi, à condition que les valeurs de 5,0 et 1,4 soient significativement différentes de $O$, il suffit que l'organisation totale ne soit pas diminuée par l'apport pour qu'il existe une ANI vraie. Celle-ci ne représenterait toutefois qu'une fraction peu significative de l'ANI mesurée. En absence de test statistique nous ne pouvons pas réellement prouver l'existence d'une ANI réelle. Selon Jenkinson et al. (1985), on doit alors admettre la possibilité d'un rôle fondamental de l'échange de pools dans I'ANI mesurée. Par conséquent, la fourniture du sol est bien indépendante de l'apport.

De même que l'azote récolté en absence d'apport, les CAU sont beaucoup plus faibles lorsqu'ils sont mesurés en $6^{\theta}$ année d'épuisement (Tableau V). La diminution des quantités d'azote facilement minéralisables au cours des années successives de récolte sans apports épigés ni fixation d'azote paraît normale dans le cas de couverts habituellement fertilisés. De la même façon, la baisse des CAU en fonction de l'épuisement résulte de la diminution de l'azote disponible à dose d'apport égale.

\section{Minéralisation brute d'azote dans le sol}

La courbe de la Figure 2, établie après 5 ans de fauche sans apport $\mathrm{N}$, donne une bonne représentation statistique des réponses à l'azote communes aux 3 prairies. Elle implique que la différence de minéralisation brute entre $P$ et $M$ (CORR) est de $200 \mathrm{~kg} \mathrm{~N}$. Elle suppose l'additivité des 2 sources d'azote minéralisé et apporté, qui est très controversée à propos des champs cultivés (Machet et al., 1987). Selon Whitehead 
(1983), Recous et al. (1988), la biomasse microbienne pourrait utiliser préférentiellement l'ammonium de l'apport alors que les végétaux supérieurs utiliseraient une plus grande partie de l'azote (nitrique) issu du sol. Ces conditions ne semblent pas réunies ici en raison d'une activité biologique et d'une nitrification limitées par l'altitude et l'acidité des sols. Au délà de la forme de l'azote minéral, l'hypothèse d'un comportement identique selon les sources peut en effet s'avérer moins fausse en prairie qu'en champs cultivés : comme l'azote apporté, l'azote minéralisé se retrouve surtout en surface par suite de la concentration de la vie active et des nutriments dans l'horizon supérieur du sol; les apports sont fréquents, et voisins des rythmes de minéralisation et de prélèvement par les plantes.

La part d'azote marqué contenue dans les parties récoltées $\left(N_{\text {dft }} \%\right)$ est de $26,6 \%$ sur les 2 pelouses. Selon le principe de la valeur A (Fried \& Dean, 1952), ce rapport représente le taux moyen de marquage du pool minéral. La minéralisation brute, supposée égale à la valeur $\mathrm{A}$, soit Val $A=$ Apport $\times\left(100-N_{\text {dff }} \%\right) / N_{\text {dff }} \%$, donne $276 \mathrm{~kg} \mathrm{~N} \cdot \mathrm{ha}^{-1} \cdot \mathrm{an}^{-1}$ pour $\mathrm{P}$ et $414 \mathrm{~kg} \mathrm{~N} / \mathrm{ha}$ pour $\mathrm{M}$. Selon cette estimation, les écarts de minéralisation brute sont seulement de $138 \mathrm{~kg} \mathrm{~N} \cdot \mathrm{ha}^{-1} \cdot \mathrm{an}^{-1}$. Le terme R's/CRU donne la part de la minéralisation brute dévolue, en absence d'apport, aux parties récoltées, soit $130(33,2 / 0,255)$ pour $P$ et $209 \mathrm{~kg} \mathrm{~N} \cdot \mathrm{ha}^{-1}(67,2 / 0,322)$ pour M. La différence entre la valeur $A$ et ce dernier terme est donnée aussi par la formule $\left(\mathrm{N}_{\mathrm{App}} . \mathrm{Cl}\right) / \mathrm{CRU}$ (Jenkinson et al., 1985), soit $\mathrm{ANI} \mathrm{I}_{m} / \mathrm{CRU}$, et vaut 146 (P) et 205 $\mathrm{kg} \mathrm{N} \cdot \mathrm{ha}^{-1} \cdot \mathrm{an}^{-1}(\mathrm{M})$. Mais les auteurs cités ont bien montré que ces quantités n'ont pas de valeur générale dans la mesure où elles dépendent du niveau d'apport marqué utilisé.

\section{CONCLUSION}

Les expériences de marquage mettent en évidence d'importants écarts entre les coefficients apparents et réels, sans qu'il soit possible de prouver l'existence d'une surminéralisation vraie d'azote du sol sous l'influence de l'apport. Dans ces conditions, on ne peut pas réfuter que le supplément d'azote issu du sol mesuré en présence d'apports soit essentiellement attribuable à l'échange de pools dans le sol. De toute façon, les mesures par marquage de l'azote récolté issu de l'engrais ou du sol sont contingentes des apports et des vitesses de minéralisation et d'organisation dans le sol. Une modélisation des flux d'azote dans le système sol-peuplement admettant une minéralisation indépendante des apports devrait permettre d'estimer les valeurs probables de ces vitesses. Encore, différentes hypothèses devraient-elles être envisagées quant aux modifications éventuelles de l'organisation en fonction des apports.

En attendant, la mesure de l'azote exporté et des coefficients apparents reste la meilleure méthode pour caractériser la fertilité de ces prairies. Ces mesures ont montré une liaison positive entre les coefficients apparents d'utilisation de l'azote disponible et l'azote récolté en l'absence d'apports. II resterait à explorer le comportement de l'azote dans les sols correspondants. Ce sera le thème d'un prochain travail.

\section{REMERCIEMENTS}

Nous remercions L. Gachon qui a favorisé l'emploi du ${ }_{15} \mathrm{~N}$ dans le laboratoire, B. Mary, de la Station d'Agronomie de Laon pour son aide à l'interprétation des résultats et l' "Intergroupe Azote" du Ministère de l'Environnement (Sretie) pour son aide.

\section{RÉFÉRENCES}

Ball P.R. (1984) Nitrogen relationships in intensively managed temperate grasslands. Plant Soil. 76, 23-33

Barraclough D., Geens E.L., Davies G.P. \& Maggs J.M. (1985) Fate of fertilizer nitrogen. Ill. The use of single and double labelled ${ }_{15} \mathrm{~N}$ ammonium nitrate to study nitrogen uptake by ryegrass. J. Soil Sci. 36, 593603

Bristow A.W., Ryden J.C. \& Whitehead D.C. (1987) The fate at several times interval of ${ }_{15} \mathrm{~N}$-labelled ammonium nitrate applied to an established grass sward. J. Soil Sci. 38, 245-254

Colbourn P. (1983) Losses of fertilizer from grass sward. In: Nitrogen Fluxes in Intensive Grassland Systems (EEC Workshop) Wageningen, 12-14 Oct. $1983,147-149$

Cuttle S.P. (1983) Aspects of nitrogen cycling in improved upland pastures. In: Nitrogen Fluxes in Intensive Grassland Systems (U.K. EEC Workshop) Wageningen, 12-14 Oct. 1983, 101-105

Dawson K.P. \& Ryden D.C. (1985) Uptake of fertilizer and soil nitrogen by ryegrass swards during spring and mid-season. Fert. Res. 6, 177-188

Fried M. \& Dean L.A. (1952) A concept concerning the measurement of available soil nutrients. Soil Sci. 73, 263-271

Jenkinson D.S., Fox R.L. \& Rayner J.H. (1985) Interactions between fertilizer nitrogen and soil nitrogen - the so-called priming effect. J. Soil Sci. 36, 425-444

Loiseau P. (1989) Contribution à une unification des lois de réponse à la fertilisation minérale azotée selon la fertilité acquise par les prairies permanentes. Fourrages 117, 3-16

Loiseau P., Jauneau A. \& Ricou G. (1984) Etudes sur le recyclage dans l'écosystème prairial. I. Influence de la conduite du pâturage sur l'activité biologique des pelouses montagnardes. Oecol. Applic. 5, 23-41 
Machet J.-M., Pierre D., Recous S. \& Remy J.-C. (1987) Signification du coefficient réel d'utilisation et conséquences pour la fertilisation azotée des cultures. C.R. Acad. Agric. Fr. 73, 39-56

Recous S., Machet J.-M. \& Mary B. (1988) The fate of labelled ${ }_{15} \mathrm{~N}$ urea and ammonium nitrate applied to a winter wheat crop. II. Plant uptake and $\mathrm{N}$ efficiency. Plant Soil, 112, 215-222

Triboi E. (1987) Recovery of mineral $N$ fertilizer in herbage and soil organic matter in grasslands of the Massif Central, France. Fert. Res. 13, 99-116
Whitehead D.C. (1983) Nitrogen dynamics in the soil under intensively managed grassland. In : Nitrogen Fluxes in Intensive Grassland Sysiems (EEC Workshop), Wageningen, Oct. 1983, 68-78

Whitehead D.C. (1984) Interactions between soil and fertilizer in the supply of nitrogen to ryegrass grown on 21 soils. J. of the Sci. of Food and Agr. 35, 10, 10671075

Whitehead D.C. \& Dawson K.P. (1984) Nitrogen, including ${ }_{15} \mathrm{~N}$ labelled fertilizer nitrogen, in components of a grass sward. J. Appl. Ecol. 21, 983-989 